\title{
Trajectory of chemotherapy for patients with EGFR wild-type advanced pulmonary adenocarcinoma: a single-institution retrospective study
}

This article was published in the following Dove Press journal:

Lung Cancer:Targets and Therapy

22 February 2017

Number of times this article has been viewed

\section{Seigo Minami \\ Yoshitaka Ogata \\ Shouichi Ihara \\ Suguru Yamamoto \\ Kiyoshi Komuta}

Department of Respiratory Medicine, Osaka Police Hospital, Osaka, Japan
Correspondence: Seigo Minami Department of Respiratory Medicine, Osaka Police Hospital, I0-3I Kitayamacho, Tennoji-ku, Osaka 543-0035, Japan $\mathrm{Tel}+8$ I 6 677| 605 I

Fax +816 677I 2838

Email seigominami@oph.gr.jp
Background: Pulmonary adenocarcinoma, recently benefited by new cytotoxic and molecularly targeted drugs, has been classified by driver mutations, such as EGFR mutations. The aim of this study was to research the proportions of patients treated with first- to third-line chemotherapy and to find influential factors for the introduction of chemotherapy and survival benefit from chemotherapy.

Materials and methods: Data were collected retrospectively on patients who met the following criteria: adenocarcinoma, diagnosed between June 2007 and March 2015 at our hospital, stage IIIB or IV, and EGFR wild type. A nonchemotherapy group of patients who did not receive chemotherapy was compared with a chemotherapy group of patients who received it. The patients who had received first- to third-line chemotherapy between June 2007 and November 2015 at our hospital were also analyzed.

Results: During the study period, 46 patients did not receive chemotherapy, while 148, 89, and 48 received first-, second- and third-line chemotherapy, respectively. As predictive factors for unlikely chemotherapy, multivariate logistic analysis detected Eastern Cooperative Oncology Group (ECOG) performance status (PS) $\geq 2$, hemoglobin $<13.2 \mathrm{~g} / \mathrm{dL}$, creatinine clearance (Ccr) $<50.4 \mathrm{~mL} / \mathrm{min}$, and $\mathrm{CRP} \geq 0.53 \mathrm{mg} / \mathrm{dL}$. As factors predicting shorter survival after chemotherapy, multivariate Cox proportional-hazard analyses detected age $\geq 75$ years, ECOG PS $\geq 2$, lower lymphocyte counts, and higher CRP for the first line; female, higher neutrophil counts, lower lymphocyte counts, reduced Ccr, hyponatremia, and shorter interval between first- and secondline chemotherapy for the second line; and age $\geq 75$ years, body mass index $<18.5 \mathrm{~kg} / \mathrm{m}^{2}$, higher neutrophil counts, lower lymphocyte counts, hyponatremia, higher lactate dehydrogenase, and higher CRP for the third line.

Conclusion: Approximately $76 \%$ of patients were treated with first-line chemotherapy. Of those patients, $61 \%$ and $34 \%$ proceeded to second- and third-line chemotherapy, respectively. For patients with poor PS, anemia, reduced Ccr, and higher CRP, it is difficult to introduce chemotherapy.

Keywords: adenocarcinoma, non-small-cell lung cancer, first-line chemotherapy, second-line chemotherapy, third-line chemotherapy, EGFR wild type

\section{Introduction}

Pulmonary adenocarcinoma is a major histological subtype of lung cancer, and has a constant tendency to increase in Japan, irrespectively of sex. Its incidence rates were $43 \%$ and $67 \%$ of all histological types of Japanese lung cancer cases during 1999-2003 in males and females, respectively. ${ }^{1}$ 
Over the last decade, in contrast to squamous cell carcinoma, treatment for adenocarcinoma has developed dramatically. This histology has benefited from a new cytotoxic antitumor drug, pemetrexed, ${ }^{2}$ and a molecularly targeted antibody - bevacizumab. ${ }^{3}$ Furthermore, adenocarcinoma has been divided into two subsets according to genetic information: adenocarcinoma harboring a driver mutation, either positive EGFR mutation ${ }^{4}$ or ALK rearrangement; ${ }^{5}$ and adenocarcinoma without these driver mutations. Nowadays, treatment strategies differ markedly between these two subsets. For the former subset, specific tyrosine-kinase inhibitors are indispensable. On the other hand, for the latter subset, cytotoxic chemotherapy remains a standard treatment. Platinumbased combination regimens with or without bevacizumab are recommended as the first-line treatment. However, almost all patients experience progression during or after first-line chemotherapy. Some of them require salvage chemotherapy.

Some survey studies have revealed a trend of patients with advanced non-small-cell lung cancer who have received first- and later-line chemotherapy. ${ }^{6-15}$ However, there is no study that has focused on patients with EGFR wild-type adenocarcinoma and followed their course of chemotherapy. Our retrospective study for adenocarcinoma with wild-type EGFR aimed to investigate 1) what the rate of patients who had received first-, second, or third-line chemotherapy was and 2) who benefited from chemotherapy.

\section{Materials and methods}

\section{Patients and study design}

This was a single-institution retrospective study. The inclusion criteria were: 1) histologically or cytologically diagnosed with pulmonary adenocarcinoma; 2) stage IIIB or IV, defined by the seventh TNM (tumor, node, metastasis) classification of lung cancer by the Union for International Cancer Control ${ }^{16}$ (staging by sixth edition of the UICC classification was reclassified according to seventh edition); (3) diagnosed between June 2007 and March 2015 at our institution; (4) EGFR wild-type status examined by LSI Medience Corporation (Tokyo, Japan) using the peptide nucleic acid-locked polymerase chain-reaction clamp method. ${ }^{17}$ The exclusion criteria were: 1) patients who were diagnosed at our hospital, but thereafter transferred to other hospitals for aggressive treatment; 2) diagnosis and introduction of any aggressive treatment were performed at another hospital, but thereafter transferred to our hospital for later-line treatment; 3) adenocarcinoma combined with other histological types; 4) immunohistochemically positive $A L K$ gene rearrangement; 5) EGFR mutations were not examined. In Japan, $E G F R$-mutation tests became covered by insurance in June
2007. Fluorescence in situ hybridization and immunohistochemistry for $A L K$ gene rearrangement were approved in April 2012 and June 2014, respectively.

1) In order to investigate predictive factors influencing introduction of systemic chemotherapy, we compared patients who had received chemotherapy (chemotherapy group) with those who had not received chemotherapy (nonchemotherapy group). 2) To find prognostic factors influencing survival after each line of chemotherapy, we extracted three cohorts of patients who had started first-, second-, or third-line regimens between June 2007 and November 2015. We excluded chemotherapies that started after December 2015. During the study period in Japan, erlotinib, pemetrexed, bevacizumab, $n a b$-paclitaxel, and nivolumab were approved for non-smallcell lung cancer in October 2007, May 2009, November 2009, February 2013, and December 2015, respectively.

Patients' background data, laboratory data, criteria and definitions of overall response rate (RR), overall survival (OS), and progression-free survival (PFS) in this study followed those of our previous studies. ${ }^{18,19}$ In this study, we added creatinine clearance estimated by Cockcroft-Gault formula as an explanatory variable at diagnosis and at the start of each chemotherapy. We added $0.2 \mathrm{mg} / \mathrm{dL}$ to serum creatinine concentrations measured by the enzymatic method, in order to adjust the difference between creatinine values measured by the Jaffe method and the enzymatic method. ${ }^{20}$ In principle, laboratory data comparing between the chemotherapy and nonchemotherapy groups were obtained on the last day before the examination leading to the confirmed diagnosis of malignancy. However, alkaline phosphatase (ALP) values were not measured before the examination in three and one cases in the chemotherapy and nonchemotherapy groups, respectively. Instead, we used the data on the nearest day after the examination in those four cases. Height could not be measured in three patients in the nonchemotherapy group, because they could not stand by themselves. Therefore, these three patients were excluded from comparison of body mass index. Data cutoff for RR, PFS, and OS was July 31, 2016. The Osaka Police Hospital ethics committee approved this study (number 501) and waived the requirement for informed consent, because our data were retrospective and deidentified.

\section{Data analysis}

Continuous, discrete, and categorical variables are expressed as mean \pm standard deviation, median (range), and frequency, respectively. Normality of distribution and homogeneity of variances were assessed by Shapiro-Wilk and $F$-tests, respectively. Comparing relative frequencies, discrete variables, and normally distributed continuous variables between two 
groups, we used $\chi^{2}$ or Fisher's exact test, Mann-Whitney $U$ test, and Student's $t$-test, respectively. Thereafter, all variables with a $P$-value $<0.2$ were included in the subsequent univariate logistic regression analysis. The laboratory data were divided by the optimal cutoff values and transformed into dichotomous variables. For receiver-operating characteristic curves and areas under the curve, cutoff points were decided as the value that gave maximal joint sensitivity and specificity. Logistic regression analysis and Cox proportionalhazard analysis were used to find factors influencing outcome variables, introduction of chemotherapy, and survival after chemotherapy, respectively. All variables with a $P$-value $<0.1$ in univariate analysis were included in the subsequent multivariate analysis. When a moderate-to-strong correlation (Pearson's correlation coefficient, $r \geq 0$.4) was observed between two laboratory data and a risk of multicollinearity was a concern, we arbitrarily excluded either of the coefficients from candidate explanatory variables in the multivariate analysis. Backward-stepwise selection based on $P$-value was used to select variables that were entered into the multivariate model. These results were expressed as odds ratios (ORs) or hazard ratios (HRs) and 95\% confidence intervals (CIs). A $P$-value $<0.05$ was statistically significant. All statistical analyses were performed with EZR (Saitama Medical Center, Jichi Medical University, Saitama, Japan), which is a graphical user interface for R (R Foundation for Statistical Computing, Vienna, Austria). ${ }^{21}$

\section{Results}

Among 194 patients who met the inclusion criteria, 148 patients were able to receive chemotherapy (chemotherapy group), but 46 could not (nonchemotherapy group). On the other hand, 62 patients were excluded from analysis, because their EGFR-mutation status was unknown. Among these 62 patients, 19 (31\%) received chemotherapy. ALK-immunohistochemistry screening was performed in 22 patients (48\%) of the nonchemotherapy group and $59(40 \%)$ of the chemotherapy group. In the chemotherapy group, 89 and 48 patients proceeded to second- and third-line chemotherapy, respectively (Figure S1).

Initially, we compared backgrounds and laboratory data between the chemotherapy and nonchemotherapy groups. Patients in the chemotherapy group were younger $(67 \pm 9.5$ vs $73.9 \pm 9.7$ years, $P<0.01)$, included a lower proportion of stage IV $(P<0.01)$, kept better Eastern Cooperative Oncology Group (ECOG) performance status (PS) $(P<0.01)$, and survived longer (median 404 vs 87 days, $P<0.01$ ) (Table 1). As for laboratory data, there were significant differences in absolute lymphocyte count $(1,721 \pm 570$ vs $1,332 \pm 562$ cells $/ \mu \mathrm{L}, P<0.01)$, hemoglobin $(13.5 \pm 1.6$ vs $11.9 \pm 1.8 \mathrm{~g} /$ $\mathrm{dL}, P<0.01)$, creatinine clearance $(59.4 \pm 18.8$ vs $46.3 \pm 21.6$ $\mathrm{mL} / \mathrm{min}, P<0.01)$, serum sodium concentration $(139 \pm 3.3$ vs $137.8 \pm 3.9, P=0.04)$, ALP $(297.6 \pm 158.9$ vs $351.9 \pm 184.7$, $P=0.04)$, and CRP $(2.1 \pm 3.4$ vs $4.8 \pm 3.5, P<0.01)$ (Table 1$)$. Table S1 presents the optimal cutoff values for binarization of laboratory data. Univariate analysis detected ten variables as factors predicting difficult introduction of chemotherapy - age $\geq 75$ years, stage IV, ECOG PS $\geq 2$, neutrophil count $\geq 8,356$ cells $/ \mu \mathrm{L}$, lymphocyte count $<1,414$ cells $/ \mu \mathrm{L}$, hemoglobin $<13.2 \mathrm{~g} / \mathrm{dL}$, sodium $<139 \mathrm{mEq} / \mathrm{L}$, creatinine

Table I Patient characteristics at diagnosis

\begin{tabular}{|c|c|c|c|}
\hline Variables & Chemotherapy & Nonchemotherapy & $P$ \\
\hline$n$ & 148 & 46 & \\
\hline \multicolumn{4}{|l|}{ Age (years) } \\
\hline Mean \pm SD & $67.0 \pm 9.5$ & $73.9 \pm 9.7$ & $<0.0 \mathrm{I}^{\mathrm{a}}$ \\
\hline \multicolumn{4}{|l|}{$\operatorname{Sex}(n)$} \\
\hline Male/female & $106 / 42$ & $32 / 14$ & $0.93^{\mathrm{b}}$ \\
\hline \multicolumn{4}{|l|}{ Smoking habits (n) } \\
\hline $\begin{array}{l}\text { Non/ex-smokers } \\
\text { vs current } \\
\text { smokers }\end{array}$ & $71 / 77$ & $27 / 19$ & $0.27^{\mathrm{b}}$ \\
\hline \multicolumn{4}{|l|}{ Staging (n) } \\
\hline IIIB/IV & $31 / 117$ & $1 / 45$ & $<0.01^{\mathrm{c}}$ \\
\hline \multicolumn{4}{|l|}{ ECOG PS $(n)^{d}$} \\
\hline $0-1 / 2 / 3 / 4$ & $12 \mid / 23 / 4 / 0$ & $11 / 16 / 15 / 4$ & $<0.0 \mathrm{I}^{\mathrm{a}}$ \\
\hline \multicolumn{4}{|l|}{ BMI $\left(\mathrm{kg} / \mathrm{m}^{2}\right)^{\mathrm{d}}$} \\
\hline Mean $\pm S D$ & $22.1 \pm 3$ & $21.5 \pm 3.4^{\mathrm{e}}$ & $0.34^{f}$ \\
\hline \multicolumn{4}{|l|}{ Charlson } \\
\hline \multicolumn{4}{|l|}{ Comorbidity Index } \\
\hline Mean \pm SD & $0.9 \pm 1.1$ & $1.1 \pm 1.4$ & $0.26^{\mathrm{a}}$ \\
\hline \multicolumn{4}{|l|}{ Overall survival } \\
\hline \multicolumn{4}{|l|}{$(\text { days })^{g}$} \\
\hline Median $(95 \% \mathrm{Cl})$ & $404(330-573)$ & $87(44-109)$ & $<0.0 \mathrm{I}^{\mathrm{h}}$ \\
\hline \multicolumn{4}{|l|}{ Laboratory data $^{d}$} \\
\hline Neutrophils (cells $/ \mu \mathrm{L}$ ) & $6,008 \pm 2,933$ & $7,106 \pm 4,090$ & $0.13^{\mathrm{a}}$ \\
\hline \multicolumn{3}{|l|}{$($ cells $/ \mu \mathrm{L})$} & $<0.01^{a}$ \\
\hline Monocytes (cells/ $\mu \mathrm{L}$ ) & $478 \pm 194$ & $555 \pm 291$ & $0.24^{\mathrm{a}}$ \\
\hline Hemoglobin (g/dL) & $13.5 \pm 1.6$ & $11.9 \pm 1.8$ & $<0.0 \mathrm{I}^{\mathrm{f}}$ \\
\hline RDW (\%) & $13.7 \pm 1.1$ & $14.0 \pm 1.6$ & $0.24^{a}$ \\
\hline $\begin{array}{l}\text { Platelets } \\
\left(\times 10^{3} \text { cells } / \mu \mathrm{L}\right)\end{array}$ & $269.2 \pm 82.1$ & $287.3 \pm 127$ & $0.84^{a}$ \\
\hline $\operatorname{Ccr}(\mathrm{mL} / \mathrm{min})$ & $59.4 \pm 18.8$ & $46.3 \pm 21.6$ & $<0.0 \mathrm{I}^{\mathrm{a}}$ \\
\hline Sodium (mEq/L) & $139 \pm 3.3$ & $137.8 \pm 3.9$ & $0.04^{\mathrm{a}}$ \\
\hline LDH (IU/L) & $291.6 \pm 333.5$ & $525.6 \pm 1358.1$ & $0.4 \mathrm{I}^{\mathrm{a}}$ \\
\hline ALP (IU/L) & $297.6 \pm 158.9$ & $351.9 \pm 184.7$ & $0.04^{\mathrm{a}}$ \\
\hline CRP (mg/dL) & $2.1 \pm 3.4$ & $4.8 \pm 3.5$ & $<0.0 \mathrm{I}^{\mathrm{a}}$ \\
\hline
\end{tabular}

Notes: a Mann-Whitney $U$ test; ' $\chi^{2}$ test; 'Fisher's exact test; ${ }^{d}$ nearest point before diagnostic examination was performed; ethree patients were excluded because height could not be measured at diagnosis; 'Student's t-test; 8from diagnosis to death or last survival confirmation; ${ }^{\text {hlog-rank test. }}$

Abbreviations: ALP, alkaline phosphatase; BMI, body mass index; $\mathrm{CCl}$, Charlson Comorbidity Index; Ccr, creatinine clearance; Cl, confidence interval; ECOG, Eastern Cooperative Oncology Group; LDH, lactate dehydrogenase; OS, overall survival; PS, performance status; RDW, red cell distribution width; SD, standard deviation. 
clearance $<50.4 \mathrm{~mL} / \mathrm{min}$, ALP $\geq 293 \mathrm{IU} / \mathrm{L}$, and CRP $\geq 0.53$ $\mathrm{mg} / \mathrm{dL}$ - while multivariate analysis extracted the four variables ECOG PS $\geq 2$ (OR 0.08, 95\% CI: 0.03-0.21; $P<0.01$ ), hemoglobin $<13.2 \mathrm{~g} / \mathrm{dL}$ (OR 0.13, 95\% CI: 0.05-0.36; $P<0.01$ ), creatinine clearance $<50.4 \mathrm{~mL} / \mathrm{min}$ (OR $0.36,95 \%$ CI: $0.15-0.9 ; P=0.03$ ), and $\mathrm{CRP} \geq 0.53 \mathrm{mg} / \mathrm{dL}$ (OR $0.33,95 \%$ CI: $0.12-0.88 ; P=0.03$ ) (Table 2).

Table 3 shows pretreatment characteristics and laboratory data at the start of first-, second-, and third-line chemotherapy, respectively. Carboplatin-based combination regimens were most frequently used in the first-line setting, while nonplatinum monotherapy regimens were used in the second- and third-line settings (Table S2). Progressive disease was always the most frequent reason for discontinuation of chemotherapy. RR, disease-control rate, and PFS gradually diminished every line (Table S3).

As factors predicting OS from the start of first-, second-, and third-line chemotherapy, univariate Cox-hazard analysis

Table 2 Univariate and multivariate logistic regression analyses of factors influencing receipt of chemotherapy

\begin{tabular}{|c|c|c|c|c|c|c|}
\hline \multirow[t]{2}{*}{ Variables } & \multicolumn{3}{|c|}{ Univariate } & \multicolumn{3}{|c|}{ Multivariate $^{a}$} \\
\hline & OR & $95 \% \mathrm{Cl}$ & $\mathbf{P}$ & OR & $95 \% \mathrm{Cl}$ & $P$ \\
\hline \multicolumn{7}{|l|}{ Age } \\
\hline $\begin{array}{l}<75 \text { vs } \geq 75 \\
\text { years }\end{array}$ & 0.35 & $0.18-0.69$ & $<0.01$ & & & \\
\hline \multicolumn{7}{|l|}{ Staging } \\
\hline IIIB vs IV & 0.08 & $0.01-0.63$ & 0.02 & & & \\
\hline \multicolumn{7}{|l|}{ ECOG PS } \\
\hline $0-1$ vs $2-4$ & 0.07 & $0.03-0.16$ & $<0.01$ & 0.08 & $0.03-0.21$ & $<0.01$ \\
\hline \multicolumn{7}{|l|}{ Neutrophils } \\
\hline $\begin{array}{l}<8,356 \text { vs } \\
\geq 8,356\end{array}$ & 0.42 & $0.19-0.93$ & 0.03 & & & \\
\hline \multicolumn{7}{|l|}{ Lymphocytes } \\
\hline \multicolumn{7}{|l|}{$($ cells $/ \mu L)$} \\
\hline $\begin{array}{l}\geq \mathrm{I}, 4 \mid 4 \text { vs } \\
<\mathrm{l}, 4 \mid 4\end{array}$ & 0.26 & $0.13-0.53$ & $<0.01$ & & & \\
\hline \multicolumn{7}{|l|}{$\begin{array}{l}\text { Hemoglobin } \\
\text { (g/dL) }\end{array}$} \\
\hline$\geq 13.2$ vs $<13.2$ & 0.13 & $0.06-0.29$ & $<0.01$ & 0.13 & $0.05-0.36$ & $<0.01$ \\
\hline \multicolumn{7}{|l|}{ Sodium } \\
\hline$\geq 139$ vs $<139$ & 0.4 & $0.21-0.74$ & $<0.01$ & & & \\
\hline \multicolumn{7}{|l|}{$\operatorname{Ccr}(\mathrm{mL} / \mathrm{min})$} \\
\hline$\geq 50.4$ vs $<50.4$ & 0.22 & $0.11-0.46$ & $<0.01$ & 0.36 & $0.15-0.9$ & 0.03 \\
\hline \multicolumn{7}{|l|}{ ALP (IU/L) } \\
\hline$<293$ vs $\geq 293$ & 0.43 & $0.22-0.84$ & 0.01 & & & \\
\hline \multicolumn{7}{|l|}{ CRP (mg/dL) } \\
\hline$<0.53$ vs $\geq 0.53$ & 0.26 & $0.11-0.55$ & $<0.01$ & 0.33 & $0.12-0.88$ & 0.03 \\
\hline
\end{tabular}

Note: ${ }^{a}$ Explanatory variables were selected by backward-stepwise selection based on $P$-values.

Abbreviations: ALP, alkaline phosphatase; Ccr, creatinine clearance; $\mathrm{Cl}$, confidence interval; ECOG, Eastern Cooperative Oncology Group; OR, odds ratio; PS, performance status. detected that the variables female, ECOG PS $\geq 2$, neutrophil count, lymphocyte count, monocyte count, creatinine clearance, sodium concentration, and CRP in first-line therapy (Table 4); age $\geq 75$ years, male, ECOG PS $\geq 2$, neutrophil count, lymphocyte count, monocyte count, hemoglobin, red cell distribution width (RDW), platelet count, creatinine clearance, sodium concentration, LDH, ALP, CRP, and interval between first and second lines in second-line therapy (Table 5); and ECOG PS $\geq 2$, neutrophil count, lymphocyte count, monocyte count, sodium concentration, LDH, ALP, CRP, and interval between first and third lines in third-line therapy (Table 6).

Subsequent multivariate Cox-hazard analysis extracted age $\geq 75$ years (HR $1.56,95 \%$ CI: $1.01-2.39 ; P=0.04$ ), ECOG PS $\geq 2$ (HR 3.22, 95\% CI: 1.98-5.24; $P<0.01$ ), lymphocyte count (HR 0.65, 95\% CI: 0.47-0.89; $P<0.01$ ), and CRP (HR 1.07, 95\% CI: $1.02-1.11 ; P<0.01)$ in first-line therapy (Table 4); female (HR 0.43, 95\% CI: 0.24-0.77; $P<0.01$ ), neutrophil count (HR 1.26, 95\% CI: 1.13-1.4; $P<0.01$ ), lymphocyte count (HR 0.42, 95\% CI: $0.26-0.67 ; P<0.01$ ), creatinine clearance (HR 0.81, 95\% CI: 0.68-0.95; $P<0.01)$,

Table 3 Pretreatment characteristics of patients who received first- to third-line chemotherapy

\begin{tabular}{|c|c|c|c|}
\hline Variables & First line & Second line & Third line \\
\hline $\mathrm{n}$ & 148 & 89 & 48 \\
\hline \multicolumn{4}{|l|}{ Age (years) } \\
\hline Mean \pm SD & $67.1 \pm 9.5$ & $66.5 \pm 9.5$ & $65.4 \pm 8.6$ \\
\hline \multicolumn{4}{|l|}{ Sex } \\
\hline Male/female & $106 / 42$ & $62 / 27$ & $33 / 15$ \\
\hline \multicolumn{4}{|l|}{ Staging ${ }^{a}$} \\
\hline$\leq \mathrm{IIIB} / \mathrm{IV}$ & $31 / 117$ & $13 / 76$ & $4 I / 7$ \\
\hline \multicolumn{4}{|l|}{ ECOG PS ${ }^{a}$} \\
\hline $0-1 / 2 / 3 / 4$ & $115 / 29 / 4 / 0$ & $66 / 19 / 4 / 0$ & $32 / 13 / 3 / 0$ \\
\hline \multicolumn{4}{|l|}{ BMI $\left(\mathrm{kg} / \mathrm{m}^{2}\right)^{\mathrm{a}}$} \\
\hline Mean \pm SD & $21.8 \pm 3.1$ & $21.9 \pm 3.1$ & $22.1 \pm 3.1$ \\
\hline \multicolumn{4}{|l|}{ Laboratory data $^{\mathrm{b}}$} \\
\hline Neutrophils (cells $/ \mu \mathrm{L}$ ) & $6,098 \pm 3,392$ & $4,803 \pm 2,29 \mid$ & $4,796 \pm 2,579$ \\
\hline Lymphocytes (cells/ $\mu \mathrm{L})$ & $1,632 \pm 597$ & $1,594 \pm 639$ & $\mathrm{I}, 59 \mid \pm 552$ \\
\hline Monocytes (cells $/ \mu \mathrm{L}$ ) & $544 \pm 272$ & $519 \pm 239$ & $508 \pm 209$ \\
\hline Hemoglobin $(g / d L)$ & $12.7 \pm 1.5$ & $11.6 \pm 1.7$ & $11.6 \pm 1.7$ \\
\hline RDW (\%) & $14 \pm 1.7$ & $14.8 \pm 2.3$ & $15.5 \pm 2.3$ \\
\hline Platelets $\left(\times 10^{3}\right.$ cells $\left./ \mu \mathrm{L}\right)$ & $269 \pm 83.1$ & $243.3 \pm 91.8$ & $241.3 \pm 78.1$ \\
\hline $\operatorname{Ccr}(\mathrm{mL} / \mathrm{min})$ & $53.2 \pm 16.5$ & $62.5 \pm 34.3$ & $61.5 \pm 15.4$ \\
\hline Sodium (mEq/L) & $138.6 \pm 3.6$ & $139.7 \pm 3.1$ & $139.4 \pm 2.8$ \\
\hline LDH (IU/L) & $309.3 \pm 470$ & $309.1 \pm 394.7$ & $262.1 \pm 113.1$ \\
\hline ALP (IU/L) & $325.8 \pm 263.8$ & $283 \pm 111.7$ & $293.2 \pm 116.4$ \\
\hline $\mathrm{CRP}(\mathrm{mg} / \mathrm{dL})$ & $3.0 \pm 4.6$ & $2.5 \pm 4.4$ & $1.8 \pm 2.2$ \\
\hline
\end{tabular}

Notes: at the start of each line of chemotherapy; ${ }^{b}$ data obtained nearest to the start of each line of chemotherapy.

Abbreviations: ALP, alkaline phosphatase; BMI, body mass index; $\mathrm{Ccr}$, creatinine clearance; ECOG, Eastern Cooperative Oncology Group; LDH, lactate dehydrogenase; PS, performance status; RDW, red cell distribution width; SD, standard deviation. 
Table 4 Univariate and multivariate Cox-hazard analysis of factors associated with overall survival after first-line chemotherapy

\begin{tabular}{|c|c|c|c|c|c|c|}
\hline \multirow[t]{2}{*}{ Factors } & \multicolumn{3}{|c|}{ Univariate } & \multicolumn{3}{|c|}{ Multivariate $^{\mathrm{a}}$} \\
\hline & HR & $95 \% \mathrm{Cl}$ & $\mathbf{P}$ & HR & $95 \% \mathrm{Cl}$ & $P$ \\
\hline \multicolumn{7}{|l|}{$\overline{\text { Age }}$} \\
\hline $\begin{array}{l}<75 \text { vs } \geq 75 \\
\text { years }\end{array}$ & 1.43 & $0.95-2.15$ & 0.09 & 1.56 & $1.01-2.39$ & 0.04 \\
\hline \multicolumn{7}{|l|}{ Sex } \\
\hline Male vs female & 1.78 & $1.16-2.74$ & $<0.01$ & & & \\
\hline \multicolumn{7}{|l|}{ ECOG PS } \\
\hline $0-1$ vs $2-4$ & 4.5 & $2.87-7.07$ & $<0.01$ & 3.22 & $1.98-5.24$ & $<0.01$ \\
\hline \multicolumn{7}{|l|}{ Stage } \\
\hline$<$ IIIB vs IV & 1.09 & $0.68-1.73$ & 0.72 & & & \\
\hline \multicolumn{7}{|l|}{ BMI $\left(\mathbf{k g} / \mathrm{m}^{2}\right)$} \\
\hline$\geq 18.5$ vs $<18.5$ & 1.01 & $0.61-1.65$ & 0.98 & & & \\
\hline \multicolumn{7}{|l|}{ Laboratory data } \\
\hline $\begin{array}{l}\text { Neutrophils } \\
\left(\times 10^{3} \text { cells } / \mu \mathrm{L}\right)^{b}\end{array}$ & 1.07 & $1.02-1.13$ & $<0.01$ & & & \\
\hline $\begin{array}{l}\text { Lymphocytes } \\
\left(\times 10^{3} \text { cells } / \mu \mathrm{L}\right)^{\mathrm{b}}\end{array}$ & 0.59 & $0.42-0.8$ & $<0.01$ & 0.65 & $0.47-0.89$ & $<0.01$ \\
\hline $\begin{array}{l}\text { Monocytes } \\
\left(\times 10^{2} \text { cells } / \mu \mathrm{L}\right)^{b}\end{array}$ & 1.16 & $1.08-1.25$ & $<0.01$ & & & \\
\hline $\begin{array}{l}\text { Hemoglobin } \\
(\mathrm{mg} / \mathrm{dL})\end{array}$ & 0.95 & $0.84-1.07$ & 0.4 & & & \\
\hline RDW (\%) & 0.9 & $0.78-1.05$ & 0.17 & & & \\
\hline $\begin{array}{l}\text { Platelets } \\
\left(\times 10^{5} \text { cells } / \mu \mathrm{L}\right)\end{array}$ & 0.87 & $0.69-1.09$ & 0.22 & & & \\
\hline $\operatorname{Ccr}(\times 10 \mathrm{~mL} / \mathrm{min})$ & 0.87 & $0.77-0.98$ & 0.02 & & & \\
\hline Sodium (mEq/L) & 0.65 & $0.43-0.98$ & 0.04 & & & \\
\hline $\mathrm{LDH}\left(\times 10^{2} \mathrm{IU} / \mathrm{L}\right)$ & 1.03 & $0.99-1.07$ & 0.12 & & & \\
\hline $\operatorname{ALP}\left(\times 10^{2} \mathrm{IU} / \mathrm{L}\right)$ & 1.06 & $0.99-1.12$ & 0.07 & & & \\
\hline CRP $(\mathrm{mg} / \mathrm{dL})$ & 1.09 & $1.06-1.13$ & $<0.01$ & 1.07 & $|.02-1.1|$ & $<0.01$ \\
\hline
\end{tabular}

Notes: ${ }^{a}$ Explanatory variables were selected by backward-stepwise selection based on $P$-value; ba significant correlation was observed between neutrophil and monocyte counts $(r=0.72,95 \% \mathrm{Cl}: 0.64-0.79 ; P<0.0 \mathrm{I})$, while no significant correlation was found between neutrophil and lymphocyte counts $(r=-0.05,95 \% \mathrm{Cl}:-0.2 \mathrm{I}$ to $0.1 \mathrm{I}$; $P=0.5 \mathrm{I})$ or between lymphocyte and monocyte counts $(r=0.03,95 \% \mathrm{Cl}:-0.13$ to $0.19 ; P=0.74$ ). Monocyte count was not selected as an explanatory variable in the multivariate analysis. Coded as I (age $\geq 75$ years, female, ECOG PS $2-4$, stage IV, BMI $<18.5 \mathrm{~kg} / \mathrm{m}^{2}$ ) and as 0 (age $<75$ years, male, ECOG PS $0-$ I, stage I-III, BMI $\left.\geq 18.5 \mathrm{~kg} / \mathrm{m}^{2}\right)$.

Abbreviations: ALP, alkaline phosphatase; BMI, body mass index; Ccr, creatinine clearance; $\mathrm{Cl}$, confidence interval; ECOG, Eastern Cooperative Oncology Group; $\mathrm{HR}$, hazard ratio; LDH, lactate dehydrogenase; PS, performance status; RDW, red cell distribution width.

sodium concentration (HR $0.24,95 \%$ CI: $0.09-0.6 ; P<0.01$ ), and interval between first- and second-line chemotherapy (HR $0.88,95 \%$ CI: $0.8-0.95 ; P<0.01$ ) in second-line therapy (Table 5); age $\geq 75$ years (HR 3.33, 95\% CI: $1.3-8.53$; $P=0.01$ ), body mass index (HR 3.26, 95\% CI: $1.27-8.36$; $P=0.01$ ), neutrophil count (HR 1.26, 95\% CI: 1.06-1.51; $P=0.01$ ), lymphocyte count (HR $0.42,95 \%$ CI: $0.19-0.92$; $P=0.03$ ), sodium concentration (HR 0.06, 95\% CI: 0.01-0.27; $P<0.01$ ), LDH (HR 1.4, 95\% CI: $1.03-1.90 ; P=0.03$ ), and CRP (HR 1.45, 95\% CI: $1.21-1.73 ; P<0.01$ ) in third-line therapy (Table 6).
Table 5 Univariate and multivariate Cox-hazard analysis of factors associated with overall survival after second-line chemotherapy

\begin{tabular}{|c|c|c|c|c|c|c|}
\hline \multirow[t]{2}{*}{ Factors } & \multicolumn{3}{|c|}{ Univariate } & \multicolumn{3}{|c|}{ Multivariate $^{a}$} \\
\hline & HR & $95 \% \mathrm{Cl}$ & $\mathbf{P}$ & HR & $95 \% \mathrm{Cl}$ & $P$ \\
\hline \multicolumn{7}{|l|}{ Age } \\
\hline $\begin{array}{l}<75 \text { vs } \geq 75 \\
\text { years }\end{array}$ & 1.93 & $1.14-3.28$ & 0.01 & & & \\
\hline \multicolumn{7}{|l|}{ Sex } \\
\hline Male vs female & 0.5 & $0.29-0.85$ & 0.01 & 0.43 & $0.24-0.77$ & $<0.01$ \\
\hline \multicolumn{7}{|c|}{ ECOG PS } \\
\hline $0-1$ vs $2-4$ & 3.68 & $2.15-6.31$ & $<0.01$ & & & \\
\hline \multicolumn{7}{|l|}{ Stage } \\
\hline$<$ IIIB vs IV & 0.72 & $0.38-1.35$ & 0.31 & & & \\
\hline \multicolumn{7}{|l|}{ BMI $\left(\mathbf{k g} / \mathbf{m}^{2}\right)$} \\
\hline$\geq 18.5$ vs $<18.5$ & 1.28 & $0.69-2.39$ & 0.43 & & & \\
\hline \multicolumn{7}{|l|}{ Laboratory data } \\
\hline $\begin{array}{l}\text { Neutrophils } \\
\left(\times 10^{3} \text { cells } / \mu \mathrm{L}\right)^{\mathrm{b}}\end{array}$ & 1.25 & I.14-I.38 & $<0.01$ & 1.26 & $1.13-1.4$ & $<0.01$ \\
\hline $\begin{array}{l}\text { Lymphocytes } \\
\left(\times 10^{3} \text { cells } / \mu \mathrm{L}\right)^{\mathrm{b}}\end{array}$ & 0.54 & $0.36-0.82$ & $<0.01$ & 0.42 & $0.26-0.67$ & $<0.01$ \\
\hline $\begin{array}{l}\text { Monocytes } \\
\left(\times 10^{2} \text { cells } / \mu \mathrm{L}\right)^{b}\end{array}$ & 1.22 & I.I-I.35 & $<0.01$ & & & \\
\hline $\begin{array}{l}\text { Hemoglobin } \\
(\mathrm{mg} / \mathrm{dL})^{\mathrm{b}}\end{array}$ & 0.83 & $0.7 \mathrm{I}-0.97$ & 0.02 & & - & \\
\hline RDW (\%) & 1.09 & $1.01-1.18$ & 0.02 & & - & \\
\hline $\begin{array}{l}\text { Platelets } \\
\left(\times 10^{5} \text { cells } / \mu \mathrm{L}\right)\end{array}$ & 1.47 & $1.12-1.92$ & $<0.01$ & & & \\
\hline $\operatorname{Ccr}(\times 10 \mathrm{~mL} / \mathrm{min})$ & 0.86 & $0.75-1$ & 0.048 & 0.81 & $0.68-0.95$ & $<0.01$ \\
\hline Sodium (mEq/L) & 0.12 & $0.05-0.27$ & $<0.01$ & 0.24 & $0.09-0.6$ & $<0.01$ \\
\hline $\mathrm{LDH}\left(\times 10^{2} \mathrm{IU} / \mathrm{L}\right)$ & I.II & $1.05-1.17$ & $<0.01$ & & & \\
\hline $\operatorname{ALP}\left(\times 10^{2} \mathrm{IU} / \mathrm{L}\right)$ & 1.43 & $1.16-1.77$ & $<0.01$ & & & \\
\hline CRP (mg/dL) & 1.13 & $1.08-1.18$ & $<0.01$ & & & \\
\hline
\end{tabular}

Interval between first- and second-line chemotherapy (months) ${ }^{c}$

$\begin{array}{llllll}0.87 & 0.8-0.94 & <0.01 & 0.88 & 0.8-0.95 & <0.01\end{array}$

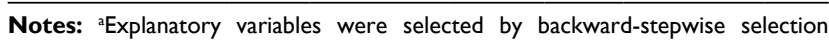
based on $P$-value. 'Significant correlations were observed between neutrophil and monocyte counts $(r=0.55,95 \% \mathrm{Cl}: 0.39-0.68 ; P<0.01)$, lymphocyte and monocyte counts $(r=0.25,95 \% \mathrm{Cl}: 0.05-0.44 ; P=0.02)$, and hemoglobin and red cell distribution width $(r=-0.35,95 \% \mathrm{Cl}:-0.52$ to $-0.15 ; P<0.01)$, while no significant correlation was found between neutrophil and lymphocyte counts $(r=0.02,95 \% \mathrm{Cl}:-0.19$ to $0.23 ; P=0.85$ ). Monocyte count was not selected as an explanatory variable in the multivariate analysis. 'From the start of first-line chemotherapy to the start of second-line chemotherapy. Coded as I (age $\geq 75$ years, female, ECOG PS $2-4$, stage IV, BMI $<18.5 \mathrm{~kg} / \mathrm{m}^{2}$ ) and as 0 (age $<75$ years, male, ECOG PS 0-I, stage I-III, BMI $\left.\geq 18.5 \mathrm{~kg} / \mathrm{m}^{2}\right)$.

Abbreviations: ALP, alkaline phosphatase; BMI, body mass index; Ccr, creatinine clearance; $\mathrm{Cl}$, confidence interval; ECOG, Eastern Cooperative Oncology Group; $\mathrm{HR}$, hazard ratio; LDH, lactate dehydrogenase; PS, performance status; RDW, red cell distribution width.

\section{Discussion}

This retrospective study is the first to clarify the trajectory of patients with advanced adenocarcinoma with $E G F R$ wild-type status. We found several factors leading to introduction of chemotherapy and longer survival after first-, second-, and third-line chemotherapy.

Our study revealed a flow of patients with adenocarcinoma with $E G F R$ wild-type status. No other study has 
Table 6 Univariate and multivariate Cox-hazard analysis of factors associated with overall survival after third-line chemotherapy

\begin{tabular}{|c|c|c|c|c|c|c|}
\hline \multirow[t]{2}{*}{ Factors } & \multicolumn{3}{|c|}{ Univariate } & \multicolumn{3}{|c|}{ Multivariate $^{a}$} \\
\hline & HR & $95 \% \mathrm{Cl}$ & $P$ & HR & $95 \% \mathrm{Cl}$ & $P$ \\
\hline \multicolumn{7}{|l|}{ Age } \\
\hline $\begin{array}{l}<75 \text { vs } \geq 75 \\
\text { years }\end{array}$ & 2.09 & $0.9-4.86$ & 0.09 & 3.33 & $1.3-8.53$ & 0.01 \\
\hline \multicolumn{7}{|l|}{ Sex } \\
\hline $\begin{array}{l}\text { Male vs } \\
\text { female }\end{array}$ & 0.75 & $0.37-\mid .51$ & 0.42 & & & \\
\hline \multicolumn{7}{|l|}{ ECOG PS } \\
\hline $0-1$ vs $2-4$ & 2.4 & $|.24-4.6|$ & $<0.01$ & & & \\
\hline \multicolumn{7}{|l|}{ Stage } \\
\hline$<$ IIIIB vs IV & 1.13 & $0.47-2.74$ & 0.78 & & & \\
\hline \multicolumn{7}{|l|}{ BMI $\left(\mathbf{k g} / \mathbf{m}^{2}\right)$} \\
\hline $\begin{array}{l}\geq 18.5 \text { vs } \\
<18.5\end{array}$ & 2.05 & $0.93-4.55$ & 0.08 & 3.26 & $1.27-8.36$ & 0.01 \\
\hline \multicolumn{7}{|l|}{ Laboratory data } \\
\hline $\begin{array}{l}\text { Neutrophils } \\
\left(\times 10^{3} \text { cells } / \mu \mathrm{L}\right)^{\mathrm{b}}\end{array}$ & 1.21 & $1.06-1.39$ & $<0.01$ & 1.26 & $|.06-1.5|$ & 0.01 \\
\hline $\begin{array}{l}\text { Lymphocytes } \\
\left(\times 10^{3} \text { cells } / \mu \mathrm{L}\right)^{\mathrm{b}}\end{array}$ & 0.27 & $0.13-0.59$ & $<0.01$ & 0.42 & $0.19-0.92$ & 0.03 \\
\hline $\begin{array}{l}\text { Monocytes }\left(\times 10^{2}\right. \\
\text { cells } / \mu \mathrm{L})^{\mathrm{b}}\end{array}$ & 1.16 & $1-1.35$ & 0.049 & & & \\
\hline $\begin{array}{l}\text { Hemoglobin } \\
(\mathrm{mg} / \mathrm{dL})\end{array}$ & 0.86 & $0.7 I-1.04$ & 0.11 & & & \\
\hline RDW (\%) & 1.04 & $0.9-1.19$ & 0.64 & & & \\
\hline $\begin{array}{l}\text { Platelets } \\
\left(\times 10^{5} \text { cells } / \mu \mathrm{L}\right)\end{array}$ & 1.3 & $0.86-1.96$ & 0.21 & & & \\
\hline $\operatorname{Ccr}(\times 10 \mathrm{~mL} /$ & & & & & & \\
\hline $\min )$ & 0.99 & $0.82-1.21$ & 0.95 & & & \\
\hline Sodium (mEq/L) & 0.28 & $0.08-0.95$ & 0.04 & 0.06 & $0.01-0.27$ & $<0.01$ \\
\hline $\mathrm{LDH}\left(\times 10^{2} \mathrm{IU} / \mathrm{L}\right)$ & 1.43 & $1.11-1.84$ & $<0.01$ & 1.4 & $1.03-1.9$ & 0.03 \\
\hline $\operatorname{ALP}\left(\times 10^{2} \mathrm{IU} / \mathrm{L}\right)$ & & & & & & \\
\hline & 1.41 & $1.03-1.94$ & 0.03 & & & \\
\hline CRP (mg/dL) & 1.31 & $1.15-1.49$ & $<0.01$ & 1.45 & $1.21-1.73$ & $<0.01$ \\
\hline $\begin{array}{l}\text { Interval between } \\
\text { chemotherapy ( } \mathrm{m}\end{array}$ & $\begin{array}{l}\text { rst- an } \\
\text { nths) }^{c}\end{array}$ & third-line & & & & \\
\hline & 0.92 & $0.86-0.99$ & 0.02 & & & \\
\hline
\end{tabular}

Notes: axplanatory variables were selected by backward-stepwise selection based on $P$-value. ${ }^{b} \mathrm{~A}$ significant correlation was observed between neutrophil and monocyte counts $(r=0.50,95 \% \mathrm{Cl}: 0.25-0.68 ; P<0.01)$, while no significant correlation was found between neutrophil and lymphocyte counts $(r=-0.11,95 \%$ $\mathrm{Cl}:-0.38$ to $0.18 ; P=0.46)$ or between lymphocyte and monocyte counts $(r=-0.03$, $95 \% \mathrm{Cl}:-0.31$ to $0.26 ; P=0.86$ ). Monocyte count was not selected as an explanatory variable in the multivariate analysis. 'From the start of first-line chemotherapy to the start of third-line chemotherapy. Coded as I (age $\geq 75$ years, female, ECOG PS $2-4$, stage IV, BMI $<18.5 \mathrm{~kg} / \mathrm{m}^{2}$ ) and as 0 (age $<75$ years, male, ECOG PS $0-$ I, stage I-III, $\left.\mathrm{BMI} \geq 18.5 \mathrm{~kg} / \mathrm{m}^{2}\right)$.

Abbreviations: ALP, alkaline phosphatase; BMI, body mass index; Ccr, creatinine clearance; $\mathrm{Cl}$, confidence interval; ECOG, Eastern Cooperative Oncology Group; $H R$, hazard ratio; LDH, lactate dehydrogenase; PS, performance status; RDW, red cell distribution width.

focused on these selected patients. 1) $76 \%$ of patients who had been diagnosed with adenocarcinoma with $E G F R$ wildtype status received chemotherapy. This proportion was higher than $67 \%$, the proportion of patients with squamous cell carcinoma during the same study period in our hospital. ${ }^{19}$ However, we are afraid that this proportion might be overestimated. We did not examine $E G F R$-mutation status intentionally in some patients with poor general condition and/or severe complications at diagnosis. Therefore, our nonchemotherapy group excluded some patients who appeared unfit for both aggressive treatment and tyrosine-kinase inhibitors at diagnosis. 2) $61 \%$ and $34 \%$ of patients who received first-line chemotherapy proceeded to second- and third-line chemotherapy, respectively. When calculating these proportions, we excluded three and three patients who remained not to progress after first- and second-line chemotherapy, respectively. These proportions were consistent with $65 \%$ and $34 \%$, those of patients with advanced pulmonary squamous cell carcinoma in our previous study. ${ }^{19}$ Therefore, the receipt rate of salvage chemotherapy was not affected by histological type. Oncologists should keep in mind that approximately a third of our patients may drop out of chemotherapy during or after the ongoing line. Therefore, we should select the best regimen at the upcoming line for each patient, rather than the best sequence of later-line regimens.

We detected some predictive markers for unlikely introduction of chemotherapy and survival benefit of each line of chemotherapy. 1) Patients with poor PS, low hemoglobin concentration, low creatinine clearance, and higher CRP value were unlikely to receive chemotherapy. Irrespective of histology, PS was a common predictive factor. ${ }^{18,19}$ Low hemoglobin was also common with squamous cell carcinoma, ${ }^{19}$ while this study additionally detected low creatinine clearance and CRP as influential factors. 2) In our previous study of squamous cell carcinoma, ${ }^{19} \mathrm{PS}$ was a common predictive factor at any time of the chemotherapeutic course. On the other hand, in this study, absolute lymphocyte count was a common predictive factor from first- to third-line chemotherapy. Ratios of neutrophils ${ }^{22-27}$ or monocytes to lymphocytes have been reported to be predictive markers for OS in advanced lung cancers. We did not assess ratios of these leukocyte differentiations as predictive markers in this study.

Our study had several limitations. It was a singleinstitution, small-sample, retrospective study. As described earlier, we did not examine EGFR-mutation status in all patients with adenocarcinoma. There might be biases for patients with unknown mutation status. We did not evaluate variables related to nutrition status, such as serum albumin concentration, because we did not measure these data routinely. Poor nutrition status may affect both introduction of chemotherapy and survival after chemotherapy. Our analyses focused exclusively on medical and physical conditions. Whether or not to start chemotherapy might depend not only on medical conditions but also on many other issues. 


\section{Conclusion}

Approximately $76 \%$ of patients with adenocarcinoma with EGFR wild-type status were treated with first-line chemotherapy. Of those patients, $61 \%$ and $34 \%$ proceeded to secondand third-line chemotherapy, respectively. Patients with poor PS, low hemoglobin concentration, low creatinine clearance, and higher CRP value tended not to receive chemotherapy. Absolute lymphocyte count was a common predictive factor from first- to third-line chemotherapy.

\section{Acknowledgments}

The authors would like to thank Son-Ho Kim, Yuki Nakatani, Narumi Noda, Kanako Nishimatsu, and Shouko Ikuta at the Department of Respiratory Medicine, Osaka Police Hospital for access to the detailed medical records of their patients and for the diagnosis, treatment, and care of their patients.

\section{Disclosure}

The authors report no conflicts of interest in this work.

\section{References}

1. Toyoda Y, Nakayama T, Ioka A, Tsukuma H. Trends in lung cancer incidence by histological type in Osaka, Japan. Jpn J Clin Oncol. 2008;38(8):534-539.

2. Hanna N, Shepherd FA, Fossella FV, et al. Randomized phase III trial of pemetrexed versus docetaxel in patients with non-small-cell lung cancer previously treated with chemotherapy. J Clin Oncol. 2004;22(9):1589-1597.

3. Sandler A, Gray R, Perry MC, et al. Paclitaxel-carboplatin alone or with bevacizumab for non-small-cell lung cancer. $N$ Engl J Med. 2006;355(24):2542-2550.

4. Lynch TJ, Bell DW, Sordella R, et al. Activating mutations in the epidermal growth factor receptor underlying responsiveness of non-small-cell lung cancer to gefitinib. N Engl J Med. 2004;350(21):2129-2139.

5. Soda M, Choi YL, Enomoto M, et al. Identification of the transforming EML4-ALK fusion gene in non-small-cell lung cancer. Nature. 2007;448(7153):561-566.

6. Asahina H, Sekine I, Horinouchi H, et al. Retrospective analysis of third-line and fourth-line chemotherapy for advanced non-small-cell lung cancer. Clin Lung Cancer. 2012;13(1):39-43.

7. Eccles BK, Geldart TR, Laurence VM, Bradley KL, Lwin MT. Experience of first- and subsequent-line systemic therapy in the treatment of non-small cell lung cancer. Ther Adv Med Oncol. 2011;3(4):163-170.

8. Girard N, Jacoulet P, Gainet M, et al. Third-line chemotherapy in advanced non-small cell lung cancer: identifying the candidates for routine practice. J Thorac Oncol. 2009;4(12):1544-1549.

9. Gridelli C, Ardizzoni A, Barni S, et al. Medical treatment choices for patients affected by advanced NSCLC in routine clinical practice: results from the Italian observational "SUN" (Survey on the IUng cancer maNagement) study. Lung Cancer. 2011;74(3):462-468.
10. Moldvay J, Rokszin G, Abonyi-Tóth Z, Katona L, Fábián K, Kóvacs G. Lung cancer drug therapy in Hungary: 3-year experience. Onco Targets Ther. 2015;8:1031-1038.

11. Murillo JR Jr, Koeller J. Chemotherapy given near the end of life by community oncologists for advanced non-small cell lung cancer. Oncologist. 2006;11(10):1095-1099.

12. Park SJ, Choi IK, Seo HY, et al. Treatment results including more than third-line chemotherapy for patients with advanced non-small cell lung cancer. Oncol Lett. 2010;1(1):51-55.

13. Reinmuth N, Payer N, Muley T, et al. Treatment and outcome of patients with metastatic NSCLC: a retrospective institution analysis of 493 patients. Respir Res. 2013;14:139.

14. Younes RN, Pereira JR, Fares AL, Gross JL. Chemotherapy beyond first-line in stage IV metastatic non-small cell lung cancer. Rev Assoc Med Bras. 2011;57(6):686-691.

15. Zietemann V, Duell T. Prevalence and effectiveness of first-, second-, and third-line systemic therapy in a cohort of unselected patients with advanced non-small cell lung cancer. Lung Cancer. 2011;73(1): 70-77.

16. Rami-Porta R, Crowley JJ, Goldstraw P. The revised TNM staging system for lung cancer. Ann Thorac Cardiovasc Surg. 2009;15(1):4-9.

17. Nagai Y, Miyazawa H, Hu Qun, et al. Genetic heterogeneity of the epidermal growth factor receptor in non-small cell lung cancer cell lines revealed by a rapid and sensitive detection system, the peptide nucleic acid-locked nucleic acid PCR clamp. Cancer Res. 2005;65(16):7276-7282.

18. Minami S, Ogata Y, Ihara S, Yamamoto S, Komuta K. Retrospective analysis of outcomes and prognostic factors of chemotherapy for smallcell lung cancer. Lung Cancer Targets Ther. 2016;7:35-44.

19. Minami S, Ogata Y, Ihara S, Yamamoto S, Komuta K. Outcomes and prognostic factors of chemotherapy for patients with locally advanced or metastatic pulmonary squamous cell carcinoma. Lung Cancer Targets Ther. 2016;7:99-110.

20. Ando Y, Minami H, Saka H, Ando M, Sakai S, Shimokata K. Adjustment of creatinine clearance improves accuracy of Calvert's formula for carboplatin dosing. Br J Cancer. 1997;76(8):1067-1071.

21. Kanda Y. Investigation of the freely available easy-to-use software 'EZR' for medical statistics. Bone Marrow Transplant. 2013;48(3):452-458.

22. Cedres S, Torrejon D, Martinez A, et al. Neutrophil to lymphocyte ratio (NLR) as an indicator of poor prognosis in stage IV non-small cell lung cancer. Clin Transl Oncol. 2012;14(11):864-869.

23. Gu XB, Tian T, Tian XJ, Zhang XJ. Prognostic significance of neutrophilto-lymphocyte ratio in non-small cell lung cancer: a meta-analysis. Sci Rep. 2015;5:12493.

24. Kos FT, Hocazade C, Kos M, et al. Assessment of prognostic value of "neutrophil to lymphocyte ratio" and "prognostic nutritional index" as a sytemic inflammatory marker in non-small cell lung cancer. Asian Pac J Cancer Prev. 2015;16(9):3997-4002.

25. Unal D, Eroglu C, Kurtul N, Oguz A, Tasdemir A. Are neutrophil/lymphocyte and platelet/lymphocyte rates in patients with non-small cell lung cancer associated with treatment response and prognosis? Asian Pac J Cancer Prev. 2013;14(9):5237-5242.

26. Yao Y, Yuan D, Liu H, Gu X, Song Y. Pretreatment neutrophil to lymphocyte ratio is associated with response to therapy and prognosis of advanced non-small cell lung cancer patients treated with firstline platinum-based chemotherapy. Cancer Immunol Immunother. 2013;62(3):471-479.

27. Yin Y, Wang J, Wang X, et al. Prognostic value of the neutrophil to lymphocyte ratio in lung cancer: a meta-analysis. Clinics (Sao Paulo). 2015;70(7):524-530. 


\section{Supplementary materials}

Table SI Receiver-operating characteristic curve analysis for optimal cutoff values of laboratory data at diagnosis for the introduction of chemotherapy

\begin{tabular}{|c|c|c|c|c|c|}
\hline Variables & Cutoff & Sensitivity & Specificity & AUC & $95 \% \mathrm{Cl}$ \\
\hline Neutrophils (cells/uL) & 8,356 & 0.28 & 0.86 & 0.58 & $0.48-0.67$ \\
\hline Lymphocytes (cells/ $\mu \mathrm{L}$ ) & $I, 4 \mid 4$ & 0.63 & 0.7 & 0.68 & $0.59-0.77$ \\
\hline Hemoglobin (g/dL) & 13.2 & 0.8 & 0.65 & 0.76 & $0.68-0.84$ \\
\hline $\operatorname{Ccr}(\mathrm{mL} / \mathrm{min})$ & 50.4 & 0.7 & 0.66 & 0.7 & $0.6 I-0.8$ \\
\hline Sodium (mEq/L) & 139 & 0.54 & 0.68 & 0.6 & $0.5-0.69$ \\
\hline ALP (IU/L) & 293 & 0.59 & 0.63 & 0.6 & $0.5-0.7$ \\
\hline CRP (mg/dL) & 0.54 & 0.78 & 0.52 & 0.66 & $0.57-0.76$ \\
\hline
\end{tabular}

Abbreviations: ALP, alkaline phosphatase; $\mathrm{AUC}$, area under the curve; $\mathrm{Ccr}$, creatinine clearance; $\mathrm{Cl}$, confidence interval.

Table S2 Regimens

\begin{tabular}{|c|c|c|c|}
\hline Regimen & First line & Second line & Third line \\
\hline $\bar{n}$ & 148 & 89 & 48 \\
\hline Platinum-based & 139 & 8 & 7 \\
\hline \multicolumn{4}{|l|}{ Platinum } \\
\hline Cisplatin-based & 37 & I & I \\
\hline Carboplatin-based & 102 & 7 & 6 \\
\hline \multicolumn{4}{|l|}{ Partner agent } \\
\hline Pemetrexed & 57 & I & I \\
\hline Paclitaxel & 66 & 5 & 2 \\
\hline Nab-paclitaxel & 2 & I & I \\
\hline SI & I & & 2 \\
\hline Gemcitabine & 3 & & \\
\hline Docetaxel & 2 & I & I \\
\hline CPTII & 1 & & \\
\hline Bevacizumab & 22 & I & \\
\hline \multicolumn{4}{|l|}{ Maintenance } \\
\hline Pemetrexed & 33 & & \\
\hline Gemcitabine & 6 & & \\
\hline Nonplatinum doublets & & 37 & 7 \\
\hline Gemcitabine + vinorelbine & & 4 & 4 \\
\hline Docetaxel + SI & & 2 & \\
\hline SI + bevacizumab & & 15 & I \\
\hline Pemetrexed + bevacizumab & & & I \\
\hline Pemetrexed + erlotinib & & 16 & \\
\hline CPTII + erlotinib & & & 1 \\
\hline Nonplatinum monotherapy & 9 & 44 & 33 \\
\hline Pemetrexed & I & 14 & 5 \\
\hline Docetaxel & I & 12 & 16 \\
\hline EGFR TKI & I & 15 & 6 \\
\hline SI & 6 & 3 & 6 \\
\hline Concurrent thoracic radiotherapy & 8 & l & \\
\hline
\end{tabular}

Abbreviation: TKI, tyrosine-kinase inhibitor. 
Table S3 Treatment response and discontinuation

\begin{tabular}{|c|c|c|c|}
\hline & First line & Second line & Third line \\
\hline$n$ & 148 & 89 & 48 \\
\hline \multicolumn{4}{|l|}{ Efficacy } \\
\hline \multicolumn{4}{|l|}{ Response } \\
\hline Complete response $(\mathrm{n})$ & 3 & 0 & I \\
\hline Partial response $(n)$ & 50 & 11 & I \\
\hline Stable disease $(n)$ & 48 & 25 & 17 \\
\hline Progressive disease (n) & 33 & 45 & 25 \\
\hline Not evaluated (n) & 14 & 8 & 4 \\
\hline Overall response rate, $\%(95 \% \mathrm{Cl})$ & $35.8(28.1-44.1)$ & $\mid 2.4(6.3-2 \mid)$ & $4.2(0.5-14.3)$ \\
\hline Disease-control rate, \% $(95 \% \mathrm{Cl})$ & $68.2(60.1-75.6)$ & $40.4(30.2-5 \mid .4)$ & $39.6(25.8-54.7)$ \\
\hline \multicolumn{4}{|l|}{ Progression-free survival (days) ${ }^{\mathrm{a}}$} \\
\hline Median $(95 \% \mathrm{Cl})$ & $157(127-167)$ & $81(50-123)$ & $92(6 I-114)$ \\
\hline \multicolumn{4}{|l|}{ Overall survival (days) } \\
\hline Median $(95 \% \mathrm{Cl})$ & $366(275-529)$ & $346(209-403)$ & $315(177-365)$ \\
\hline \multicolumn{4}{|l|}{ Reasons of discontinuation (n) } \\
\hline Progressive disease & 72 & 53 & 28 \\
\hline Completion of predefined courses & 20 & 3 & 3 \\
\hline Adverse effects & 27 & 14 & 5 \\
\hline Patient refusals & 2 & 3 & 1 \\
\hline Transfer to other hospitals & 1 & 1 & 0 \\
\hline Cancer-related deteriorated condition & 19 & 11 & 7 \\
\hline Comorbidity-related deteriorated condition & 6 & 1 & 3 \\
\hline Ongoing & 1 & 3 & 1 \\
\hline
\end{tabular}

Note: ${ }^{a}$ From initiation of first-, second-, or third-line chemotherapy.

Abbreviation: $\mathrm{Cl}$, confidence interval. 
194 Ad, no driver mutation, diagnosed from June 2007 until March 2015 at Osaka Police Hospital

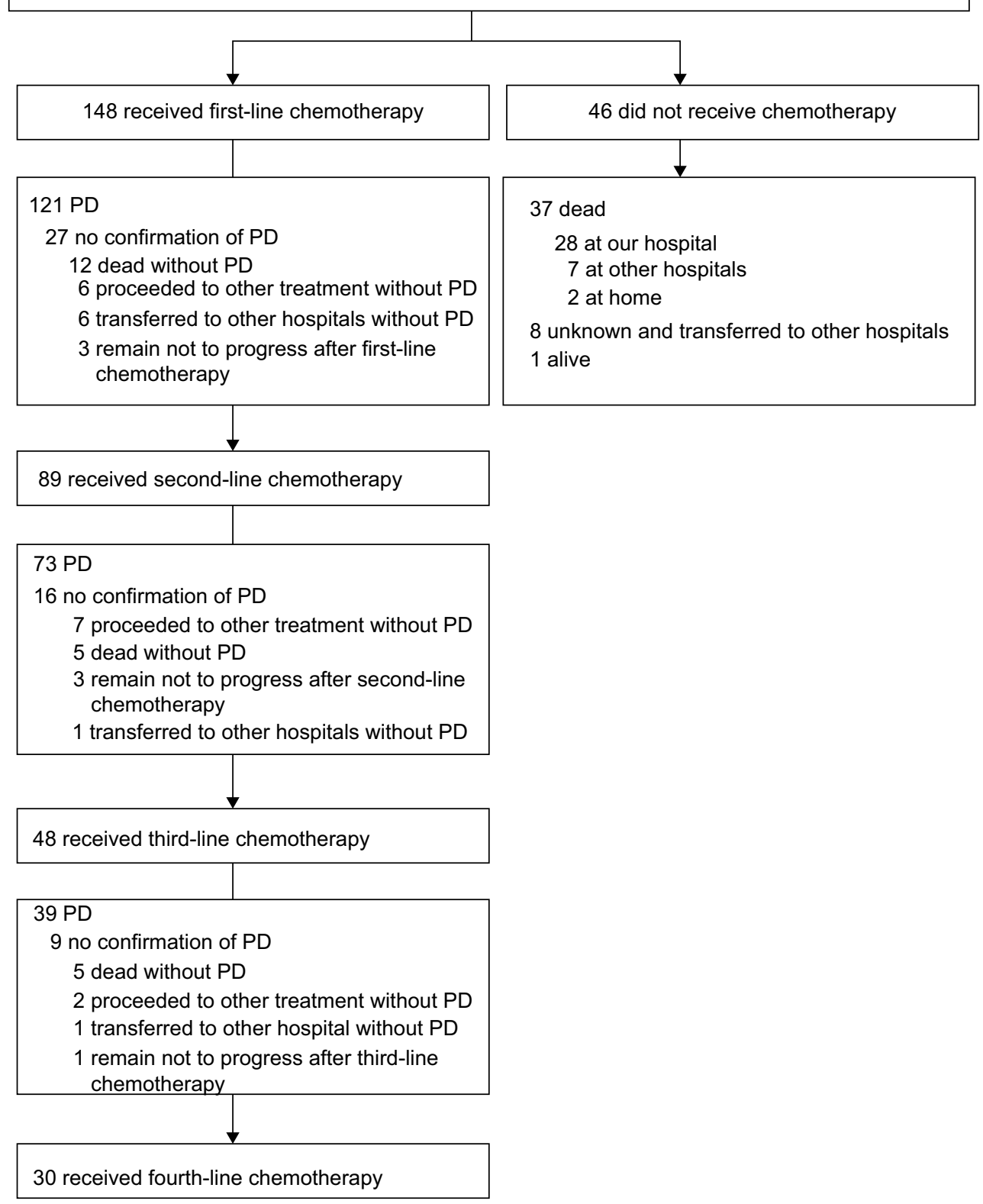

Figure SI Patient flowchart.

Abbreviations: Ad, adenocarcinoma; PD, progressive disease.

Lung Cancer: Targets and Therapy

\section{Publish your work in this journal}

Lung Cancer: Targets and Therapy is an international, peer-reviewed, open access journal focusing on lung cancer research, identification of therapeutic targets and the optimal use of preventative and integrated treatment interventions to achieve improved outcomes, enhanced survival and quality of life for the cancer patient. Specific topics covered in the journal include: Epidemiology, detection and screening; Cellular research and biomarkers; Identification of biotargets and agents with novel

mechanisms of action; Optimal clinical use of existing anticancer agents, including combination therapies; Radiation and surgery; Palliative care; Patient adherence, quality of life, satisfaction; Health economic evaluations. The manuscript management system is completely online and includes a very quick and fair peer-review system. Visit http://www.dovepress.com/testimonials.php to read real quotes from published authors.

Submit your manuscript here: https://www.dovepress.com/lung-cancer-targets--therapy-journal 